\title{
Emotions in motion: Impact of emotion understanding on children's peer action
} coordination

\author{
Karine M. P. Viana \\ Department of Psychology, University of Oslo, Oslo, Norway \\ Imac M. Zambrana \\ Department of Special Needs Education, University of Oslo, Norway \\ The Norwegian Center for Child Behavioral Development, Oslo, Norway \\ Evalill B. Karevold and Francisco Pons \\ Department of Psychology, University of Oslo, Oslo, Norway \\ Correspondence: \\ Karine Viana \\ karine.viana@psykologi.uio.no \\ Postbox 1094 Blindern 0317 Oslo \\ +4795330429 (Cell phone)
}

Keywords: Emotion understanding; Peer action coordination; Problem-solving; Children

4818 words

Date of re-submission: $22^{\text {nd }}$ of July 2019 


\title{
Emotions in motion: Impact of emotion understanding on children's peer action
}

\section{coordination}

\begin{abstract}
Peer action coordination has been often studied in terms of its underlying cognitive mechanisms, and little is known about its emotional processes. The aim of the present study was to investigate the extent to which children's emotion understanding explains their coordination of actions with a peer in a cooperative sensorimotor problem-solving task. Sixtyeight 5- to 9-year-old children were assessed for their emotion understanding with the Test of Emotion Comprehension (TEC) and for their problem-solving capacities with a sensorimotor task in an individual setting (individual sensorimotor skills) and in a cooperative setting (peer action coordination). The results showed that higher levels of emotion understanding significantly explained greater peer action coordination, even when controlling for age, gender and the child's individual sensorimotor skills. The findings point to the existence of emotional mechanisms - more specifically the role of emotion understanding - underlying successful coordination of actions in peer interaction. Theoretical and educational implications of having emotion understanding abilities for coordinating actions with others are discussed.
\end{abstract}

Keywords: Emotion understanding; Peer action coordination; Problem-solving; Children 


\section{Introduction}

Children's everyday social life demands that they align their behaviour and thoughts, both motion- and emotion-wise, with the behaviour and thoughts of others. Although this appears to demand an understanding of emotions, peer action coordination and emotion understanding have traditionally been studied separately. Action coordination involves the ability to couple relevant perceptual information, such as coordinating vision and grasping, and to integrate this with interdependent body movements, such as synchronizing the actions of separate limbs and coordinating sensorimotor behaviours with a partner (Getchell, 2006). This phenomenon has also been called "joint action", "interpersonal coordination" and "social coordination" (Eckerman \& Peterman, 2001; Michael, 2011). Because the present study focuses on peer interaction in a sensorimotor task, the term "peer action coordination" will be used and defined as an activity where two or more individuals, by playing complementary roles, adjust and align their sensorimotor actions to achieve a common goal (Grüeneisen, Wyman, \& Tomasello, 2015).

Cognitive processes essential for peer action coordination, such as representation sharing, joint attention and intention attribution in infancy and toddlerhood ([Blinded for reviewers]; Lucena \& Pedrosa, 2014;Tomasello, 2000; Warneken, Chen, \& Tomasello, 2006), as well as the ability to plan an action by taking the other's perspective into account ahead of time in middle childhood (Meyer, Bekkering, Haartsen, Stapel, \& Hunnius, 2015), have been studied extensively in the past years (Sebanz, Bekkering, \& Knoblich, 2006). In one classic study on coordination of actions in school-aged children, Doise and Mugny (1984) showed that 5- to 6-year-old children were unsuccessful in coordinating their movements in both the individual and the cooperative conditions, whereas 6- to 7-year-old children benefitted from the cooperative condition and succeeded equally well in both conditions. Satta, FerrariToniolo, Visco-Comandini, Caminiti, and Battaglia-Mayer (2017) also found that children's 
peer action coordination seemingly improved with age, with 9-year-old children performing similar to adults. This development might be related to maturation of motor skills, but it might also be a result of social interaction, a so called "cooperation benefit", as well as a notable development in inhibitory control skills. In addition, some studies have started to show that Theory of Mind (ToM) - the ability to ascribe mental states to self and others, consequently enabling the explanation, prediction and manipulation of others' actions and representations (Wellman, 2018) - is positively related to peer action coordination (e.g., Curry \& Chesters, 2012; Grüeneisen et al., 2015). However, there is fairly little knowledge on the degree to which understanding emotions, which is the affective side of ToM (e.g., Pons \& Harris, in press; Sprung, Münch, Harris, Ebesutani, \& Hofmann, 2015) can particularly impact peer action coordination.

According to Castro, Cheng, Halberstadt, and Gruehn (2016), emotion understanding comprises emotion recognition (recognizing and labelling emotions) and emotion knowledge, which is related to information one has about emotions (e.g., causes, consequences, cultural rules, etc.). Emotion understanding is therefore the ability to recognize and understand the nature, causes and consequences of emotions, its main function being to describe, explain, predict and change emotions in the self and others (for a review, see Pons \& Harris, in press). Pons, Harris, and de Rosnay (2004) have identified three qualitative hierarchical stages in the development of emotion understanding during childhood: external (2-3 to 4-5 years), mental (5-6 to 7-8 years), and reflective (8-9 to $10-11$ years). For instance, between 2-3 to $4-5$ years, children become able to recognize basic emotions and to understand the impact of external causes and desires on emotions. From about 5 to 7 years of age they begin to understand the difference between expressed and felt emotions and the impact of beliefs and memories on emotions. But it is only from around 8 to 10 years of age that they become able to understand moral and mixed emotions, and the possibility to regulate emotions by the mean of 
psychological strategies. Despite individual and cultural variations in emotion understanding development, this hierarchical organization has been found across different cultures (for a review, see Tang et al., 2018). Recent studies have also indicated that children's emotion understanding is an important predictor of their psychological well-being (e.g. Bender, Pons, Harris, Esbjørn, \& Reinholdt-Dunne, 2015), social competences (e.g. Trentacosta \& Fine, 2010), and school achievement (e.g., Voltmer \& Von Salisch, 2017).

Even though the relation between emotion understanding and peer relations has been studied in terms of, for example, popularity (e.g., Slaughter, Imuta, Peterson, \& Henry, 2015), and pro-social behaviour (e.g., Caputi, Lecce, Pagnin, \& Barnejee, 2012), it is still unclear whether emotion understanding can be one of the potential mechanisms explaining peer action coordination in the context of cooperative sensorimotor problem-solving tasks (e.g. Vesper et al., 2017;[Blinded for review]). This knowledge gap needs to be addressed because our everyday social life demands very often that we align our behaviour and thoughts with the behaviour and thoughts of others, for example, with peers in school settings. Mehu, Grammer, and Dunbar (2007) showed that cooperative individuals display higher levels of positive emotions than non-cooperators and that emotional expressivity is an evident signal of cooperativeness. Sharing emotions with others gives motivational cues that facilitate the initiation and maintenance of peer action coordination (Vesper et al., 2017). Noteworthy, so far no studies have assessed the role of emotion understanding for the success of peer action coordination.

Thus, investigating the relation between emotion understanding and peer action coordination among school age children is relevant because: 1) to coordinate actions with others is an essential part of school activities and is strongly related to important social competencies in middle childhood, such as cooperation and helping behaviours (e.g., Cirelli, Einarson, \& Trainor, 2014); 2) research has frequently focused on cognitive processes 
underlying peer action coordination, and although some studies have pointed out that emotions might play a role in action coordination (e.g., Reschke, Walle, \& Dukes, 2017), the particular contribution of emotion understanding for performing coordinated actions with peers has not been investigated .The present study can therefore advance our knowledge on the emotional mechanisms underlying peer action coordination by building a bridge between children's understanding of emotions and their capacities to coordinate their actions with peers.

\section{The present study}

This study examines the impact of children's emotion understanding on their capacity to coordinate their actions in a cooperative sensorimotor problem-solving task. Our main hypothesis is that children's emotion understanding will have a significant impact on their peer action coordination performance, even when controlling for their age, gender and individual sensorimotor skills. This is predicted due to the social nature of the cooperative condition, which most likely requires the ability to understand emotions and could therefore facilitate coordination (Vesper et al., 2017).

\section{Method}

\section{Participants}

Based on previous studies investigating the relations between emotions and action coordination (e.g., Satta et al., 2017), sample size was estimated with G*Power 3.1.9.2. on the basis of 68 participants to detect medium to large effects $(f=.15)$ at power $(0.8)$ and $\alpha=.05$ to test our main hypothesis. After the project was approved by the Norwegian Social Science Data and the Ethic Committee in Brazil, we contacted 120 parents through two middle-class private schools in Recife (Brazil). The parents of 90 children received information on the study aims and procedures and signed a consent form giving their permission for us to ask 
their children to participate. Subsequently, all children invited agreed to participate in the study.

To avoid floor and ceiling effects, children who did not succeed on the simplest item $(n=14)$ or achieved the maximum score $(n=8)$ in the individual condition were excluded from the sample. The final sample comprised 68 typically developing children ( 32 boys; 36 girls) between 5 years 7 months and 9 years 8 months $(M=90.57$ months; $S D=13.36$ months) with Portuguese as their native language. Children were divided into two age groups ( $n=34$ in the younger group: $5 y 7 m-7 y 5 m ; n=34$ in the older group: $7 y 6 m-9 y 8 m$ ). Because asymmetry in knowledge and gender might create competitive rather than collaborative relationships (e.g., Sommet, Darnon, \& Butera, 2015), the dyads consisted of children of the same gender, of similar age, from the same classroom, and with similar individual sensorimotor performance and scores on the emotion understanding test. Information from the children's ranking of their friends in the classroom was also used when composing the dyads in order to ensure that the children were neither best friends nor not friends. This aimed to promote similar opportunities to cooperate, thus avoiding potential advantages of interacting with a best friend (well-known partner) and reducing the disadvantage of getting into social conflicts when interacting with a disliked classmate (e.g., Kuhnert, Begeer, Fink, \& de Rosnay, 2017).

\section{Procedure, tasks and scoring}

The data collection consisted of three sessions conducted at the children's schools: 1) individual condition of the sensorimotor task; 2) individual assessment on the Test of Emotion Comprehension (Pons \& Harris, 2000); and 3) the cooperative condition of the sensorimotor task. The duration of each session was approximately 10 minutes, with an average interval of 15 days between sessions. 


\section{Sensorimotor problem-solving task}

Children were first tested individually and then in a cooperative condition in a task called the "labyrinth ball game", in which they had to balance a ball around different holes using two adjusting knobs to tilt the board (see Figure 1). The task requires the coordination of actions similar to the game used in the studies conducted by Doise and Mugny (1984), and it is suitable to be played by a single child from around the age of 6 , as well as by two children at the same time. When performing the task alone, the child had to use both hands, one on each knob, to guide the steel ball through the maze. When the child played with a partner, the two children held one knob each and they had to coordinate their actions and perspectives simultaneously. The first and the second levels of the game used the same board design, but they had five and eight holes, respectively; the third and the fourth levels had a more complex labyrinth design than the first two levels, with 11 and 14 holes, respectively. In total, the game had 38 holes. Children were allowed to have maximum five trials in each level.

[Figure 1 near here]

Task performance represented how close to the end of the labyrinth the children could get. In the individual condition, this is referred to as individual sensorimotor performance, whereas in the cooperative condition it is called peer action coordination performance. The scoring method was the same in both conditions. First, we calculated the sum of the number of the last hole reached in each level, ranging from zero to 38. In the cooperative condition, all children played the four levels, but in the individual condition, children would stop playing the game when they failed to complete one of the levels. To make the two conditions comparable, we calculated the ratio of the last hole reached in each condition by dividing the child's score by 38 (the total number of holes in the game). Thus, the individual sensorimotor performance and the peer action coordination performance could vary from zero to one. 


\section{Test of Emotion Comprehension (TEC)}

The Test of Emotion Comprehension (Pons \& Harris, 2000) is appropriate for the goal of the present study as it assesses both aspects of emotion understanding, i.e., emotion recognition an emotion knowledge (Castro, Cheng, Halberstadt, \& Gruehn, 2016). The test consists of a picture book containing cartoon scenarios with stories and four possible emotional outcomes (two positive: happy and alright, and two negative: scared, angry or sad). These outcomes are represented as the facial expressions of the story protagonist, which are left blank in the scenario itself. After the experimenter read the story for each scenario, the children were asked to attribute an emotion to the story protagonist(s) by pointing to the most appropriate of the emotional outcomes. The TEC assesses nine different core components of emotion understanding: recognition of basic emotions (five items), understanding the impact of situational variations on emotions (five items), understanding desire-based emotions (four items), understanding the impact of beliefs on emotions (one item), understanding the impact of memories on emotions (one item), understanding the control of the expression of emotions (one item), understanding the regulation of the experience of emotions by means of reflective psychological strategies (one item), understanding the mixed nature of emotions (one item), and understanding moral and reflective emotions (two items). Children received one point for each correct item, with a final score ranging from zero to 21 . The TEC has been translated into 27 languages, including Portuguese, and has shown good test-retest reliability, as well as concurrent, criterion and construct validity (for a recent review, see Pons \& Harris, in press).

\section{Results}

\section{Preliminary analyses}

SPSS Statistics 22.0 was used for all analyses in the present study and Cohen's kappa coefficient was used to calculate effect size. An analysis of variance Age X Gender indicated a significant and large effect of age on the TEC scores $\left(F(1,64)=16.10, p<.001, \eta^{2}=.20\right)$ but 
no significant effect of gender $\left(F(1,64)=1.90, p<.17, \eta^{2}=.03\right)$ or interaction between age and gender $\left(F(1,64)=0.007, p<.93, \eta^{2}=.00\right)$. Regardless of their gender, older children $(\mathrm{M}$ $=18.4, \mathrm{SD}=1.60)$ had better scores on the TEC than younger children $\operatorname{did}(\mathrm{M}=16.9, \mathrm{SD}=$ 1.51). A mixed between-within subject analysis of variance Age X Gender X Condition showed a significant medium effect of age $\left(F(1,64)=7.6, p=0.01, \eta^{2}=.11\right)$, a significant large effect of condition $\left(F(1,64)=158.9, p<0.01, \eta^{2}=.71\right)$, and no significant effect of gender on children's performance on the sensorimotor task $\left(F(1,64)=3.61, p<.06, \eta^{2}=.05\right)$. Older children $(M=0.70, S D=0.2)$ had higher performance than younger children $\operatorname{did}(M=$ $0.61, S D=0.2$ ), whatever the condition of the task and regardless their gender. Children had also higher performance in the cooperative condition $(M=0.81, S D=0.14)$ than in the individual condition $(M=0.50, S D=0.25)$, regardless of their age and gender. There was also a significant medium interaction effect between age and condition $\left(F(1,64)=3.8, p<0.05, \eta^{2}\right.$ $=.06)$, suggesting that older children did better on both the individual and cooperative condition, and they progressed more in the game than younger children did.

\section{Links between emotion understanding, individual sensorimotor skills and peer action}

\section{coordination}

Correlation analysis showed a significant positive and rather large $(r=.46, p<.000)$ correlation between children's emotion understanding and their peer action coordination performance in the cooperative condition, whereas no significant correlation was found between the emotion understanding and performance in the individual condition $(r=.07, p<$ .55). Moreover, children's sensorimotor performance in the individual condition was significantly positively associated (medium size) with their performance in the cooperative condition $(r=.34, p<.004)$.

Table 1 shows a summary of the predictors of children's peer action coordination performance tested through a hierarchical regression analysis. Age, gender and sensorimotor 
performance in the individual condition were entered at Step 1, explaining 38\% $\left(R^{2}=.38\right)$ of the variance in children's coordination of actions in the cooperative condition. After the TEC scores were entered at Step 2, the model explained in total $43 \%\left(R^{2}=.43\right)$ of the variance in the dependent variable $(F(4,63)=12.91, p<.000)$. Emotion understanding explained significantly an additional $6 \%$ of the variance in peer action coordination after controlling for age, gender and sensorimotor performance in the individual condition ( $\mathrm{R}$ squared change $=$ .06 , $\mathrm{F}$ change $(1,63)=6.06, p<.017)$. In the final model, age $(\beta=.38, p<.001)$, individual sensorimotor performance $(\beta=.28, p<.006)$ and emotion understanding $(\beta=.26, p<.017)$ were statistically significant in explaining peer coordination of action in the cooperative condition.

[Table 1 near here]

\section{Discussion}

The main goal of this study was to examine the impact of children's emotion understanding on their capacity to coordinate their actions in a cooperative sensorimotor problem-solving task. Our hypothesis was confirmed as the findings indicated a significant impact of children's ability to understand emotion and their performance in coordinating actions with a partner, beyond and above the effect of age, gender and their individual sensorimotor skills. First, we found that the impact of age on emotion understanding replicates findings from previous studies showing that the understanding of emotions develops over time (e.g., Pons \& Harris, in press for recent review). The fact that older children (7-9 years of age) performed better than younger ones (5-7 years of age) in the sensorimotor problem is also in line with earlier findings demonstrating that the ability to control movements in bimanual coordination increases significantly from 5 to 9 years of age (e.g., Satta et al., 2017). The improvement in the performance when children played the game together with a partner also corroborates findings from previous studies showing cooperation benefits across a wide range 
of coordination tasks (Doise \& Mugny, 1984; ([Blinded for review]; Zapiti \& Psaltis, 2012). The fact that older children progressed more on the task than younger children were also found by ([Blinded for review]) when they assessed children's ability to coordinate spatial perspectives. This could be related to the higher reliance on more advanced social and linguistic abilities in the cooperative setting, as well as on children's capacity to choose appropriate complementary actions at an appropriate time, making cooperation even more dependent on inhibitory control abilities (Satta et al., 2017).

Based on earlier findings, the impact of age and the individual sensorimotor skills on peer action coordination was therefore expected (Doise \& Mugny, 1984; Satta et al., 2017). However, our study is the first to show that emotion understanding is also a significant predictor of school-age children's peer action coordination, even when age, gender and individual sensorimotor skills are considered. Theoretically, the findings point to emotion understanding as one of the mechanisms promoting better coordination of actions in peer interaction. First, they support previous empirical studies that demonstrated that ToM has positive implications for coordinating actions with others (Curry \& Chesters, 2012; Grüeneisen et al., 2015). Second, they expand these earlier results by showing, beyond falsebelief and perspective-taking competences, the role of understanding the emotional side of the mind in peer action coordination. Consequently, the results suggest that understanding emotion goes beyond conceptual knowledge and brings positive implications for peer interaction in the context of cooperative sensorimotor task. Considering also that the task used in this study has some features that resemble school activities - i.e. the children could communicate freely, they were engaged in a game-playing task, and they were playing with classmates - the findings have educational implication in terms of the relevance of emotion understanding abilities in cooperative tasks in school settings. 


\section{Limitations and future directions}

Some limitations and prospects should be recognized. First, the small sample size of the study might have influenced the fact that we did not find a positive relationship between emotion understanding and individual sensorimotor skills. Alternatively, it could also be that although playing the game on one's own is fun and exciting, and potentially elicits different types of emotions, we are in fact more prone to talk and make sense of our own and others' emotions in social interaction than in individual contexts. Future studies with a larger sample size can better address this issue. Second, the cross-sectional nature of the design did not allow interpretation regarding the direction of the relation. Longitudinal and training approach in kindergartens and schools could address this issue. For instance, engaging children in training that helps them develop their understanding of emotional states, such as talking about emotions (e.g., Sprung et al., 2015) can potentially contribute to making them more proficient in everyday peer coordination in school settings, where they have to coordinate actions and different emotional perspectives Third, our study assessed only symmetrical dyads. It would be also interesting to analyse whether the same results would be obtained if the dyads were composed asymmetrically (e.g., cross-gender dyads, adult-child interaction and children with different levels of emotion understanding and sensorimotor skills) in order to have a clearer picture with regard to in which interactional context emotion understanding has positive implication for action coordination. Fourth, combining emotional measures with cognitive measures, such as inhibitory control and planning skills, would be relevant to provide information on the specific contribution of emotion understanding for peer action coordination when other cognitive skills are taken into consideration. Last, but not least, we did not analyse more qualitatively how children interacted while playing the game, and how they possibly used their emotion understanding abilities in the cooperative task. It would be highly relevant to examine whether the abilities to share and regulate emotions while playing 
the game with a partner mediate or moderate the impact of emotion understanding on peer action coordination performance.

In conclusion, motion and emotion can be acknowledged as two interrelated domains, as the synchronisation of our movements with the movements of others does seem to be influenced by the capacity to comprehend emotions. We would suggest that not only body and mind move together in joint actions (Sebanz et al., 2006), but that body, mind and emotions work together to perform successful coordination of actions. It seems that the better we know about our own and others' emotions, the better we coordinate what our bodies do and perceive, suggesting that, although not sufficient, the ability to understand emotions might be necessary for action and perception coordination in peer interaction.

\section{Acknowledgments}

The authors thank the children for their participation in this project and the parents who authorized their participation; Carina Pessoa Santos for helping with data collection; and participating schools for giving us access to their facilities.

\section{Conflict of interest statement}

The authors declare that the research was conducted in the absence of any commercial or financial relationships that could be construed as a potential conflict of interest

\section{Funding}

This work was supported by Lånekassen - The Norwegian State Educational Loan Fund -as part of the Quota Scheme Program, which supports students from developing countries.

\section{References}

Bender, P. K., Pons, F., Harris, P. L, Esbjørn, B. H, \& Reinholdt-Dunne, M. L. (2015). Emotion understanding in clinically anxious children: A preliminary investigation. Frontiers in 
Psychology, 6, 1-10. doi:10.3389/fpsyg.2015.01916

Caputi, M., Lecce, S., Pagnin, A., \& Banerjee, R. (2012). Longitudinal effects of theory of mind on later peer relations: The role of prosocial behavior. Developmental Psychology, 48(1), 257-270. doi: 10.1037/a0025402

Castro, V. L., Cheng, Y., Halberstadt, A. G., \& Grühn, D. (2016). EUReKA! A Conceptual Model of Emotion Understanding. Emotion Review, 8(3), 258-268. doi:10.1177/1754073915580601

Cirelli, L. K., Einarson, K. M., \& Trainor, L. J. (2014). Interpersonal synchrony increases prosocial behavior in infants. Developmental Science, 17(6), 1003-1011. doi:10.1111/desc.12193

Curry, O., \& Chesters, M. J. (2012). 'Putting ourselves in the other fellow's shoes': The role of 'theory of mind' in solving coordination problems. Journal of Cognition and Culture, 12, 147-159. doi:10.1163/156853712X633974

Doise, W., \& Mugny, G. (1984). The social development of the intellect. Oxford, UK: Pergamon Press.

Eckerman, C. O., \& Peterman, K. (2001). Peers and infant social/ communicative development. In G. Bremner \& A. Fogel (Eds.), Blackwell handbook of infant development (pp. 326-350). Oxford, UK: Blackwell.

Getchell, N. (2006). Age and task-related differences in timing stability, consistency, and natural frequency of children's rhythmic, motor coordination. Developmental Psychobiology, 48(8), 675-685. doi:10.1002/dev.20186

Grüneisen, S., Wyman, E., \& Tomasello, M. (2015). "I know you don't know I know..." Children use second-order false-belief reasoning for peer coordination. Child Development, 86(1), 287-293. doi:10.1111/cdev.12264

Kuhnert, R. L., Begeer, S., Fink, E., \& de Rosnay, M. (2017). Gender-differentiated effects of 
theory of mind, emotion understanding, and social preference on prosocial behavior development: a longitudinal study. Journal of Experimental Child Psychology, 154, 13-27. doi:10.1016/j.jecp.2016.10.001.

Lucena, J. M. F., \& Pedrosa, M. I. (2014). Stability and change in the construction of shared routines in playgroups. Psicologia Reflexão \& Crítica, 27(3), 556-563. doi:10.1590/1678-7153.201427318

Mehu, M., Grammer, K., \& Dunbar, R. I. (2007). Smiles when sharing. Evolution and Human Behavior, 28, 415-422. doi:10.1016/j.evolhumbehav.2007.05.010

Meyer, M., Bekkering, H., Haartsen, R., Stapel, J. C., \& Hunnius, S. (2015). The role of action prediction and inhibitory control for joint action coordination in toddlers. Journal of Experimental Child Psychology, 139, 203-220. doi:10.1016/j.jecp.2015.06.005

Michael, J. (2011). Shared emotions and joint action. Review of Philosophy and Psychology, 2(2), 355-373. doi:10.1007/s13164-011-0055-2

Pons, F., \& Harris, P. L. (2000). Test of emotion comprehension (TEC). Oxford, UK: Oxford University.

Pons, F., \& Harris, P. L. (in press). Children's understanding of emotions or the “error" of Pascal. In V. LoBue, K. Perez-Edgar, \& K. Buss (Eds.), Handbook of emotional development.

Pons, F., Harris, P. L., \& de Rosnay, M. (2004). Emotion comprehension between 3 and 11 years: Developmental periods and hierarchical organization. European Journal of Developmental Psychology, 1(2), 127-152. doi:10.1080/17405620344000022

Reschke, P. J., Walle, E. A., \& Dukes, D. (2017). Interpersonal development in infancy: the interconnectedness of emotion understanding and social cognition. Child Development Perspectives, 11(3), 178-183. doi:10.1111/cdep.12230 
Satta, E., Ferrari-Toniolo, S., Visco-Comandini, F., Caminiti, R., \& Battaglia-Mayer, A. (2017). Development of motor coordination during joint action in mid-childhood. Neuropsychologia, 105, 111-122. doi:10.1016/j.neuropsychologia.2017.04.027

Sebanz, N., Bekkering, H., \& Knoblich, G. (2006). Joint action: bodies and minds moving together. Trends in Cognitive Sciences, 10(2), 70-76. doi:10.1016/j.tics.2005.12.009

Slaughter, V., Imuta, K., Peterson, C. C, \& Henry, J. D. (2015). Meta-analysis of theory of mind and peer popularity in the preschool and early school years. Child Development, 86(4), 1159-1174. doi:10.1111/cdev.12372

Sommet, N., Darnon, C., \& Butera, F. (2015). To confirm or to conform? Performance goals as a regulator of conflict with more competent others. Journal of Educational Psychology, 107, 580-598. doi:10.1037/a0037240

Sprung, M., Münch, H. M., Harris, P. L., Ebesutanid C., \& Hofmann, S. G. (2015). Children's emotion understanding: A meta-analysis of training studies. Developmental Review, 37, 41-65. doi:10.1016/j.dr.2015.05.001

Tang, Y., Harris, P., Pons, F., Zou, H., Zhang, W., \& Xu, Q. (2018). The understanding of emotion among young Chinese children. International Journal of Behavioral Development, 1-6. doi:10.1177/0165025417741366

Tomasello, M. (2000). Culture and cognitive development. Current Directions in Psychological Science, 9, 37-40. Retrieved from http://journals.sagepub.com/doi/10.1111/1467-8721.00056

Trentacosta, C. J., \& Fine, S. E. (2010). Emotion knowledge, social competence, and behavior problems in childhood and adolescence: A meta-analytic review. Social Development, 19(1), 1-29. doi:10.1111/j.1467-9507.2009.00543.x

Vesper, C., Abramova, E., Bütepage, J., Ciardo, F., Crossey, B., Effenberg, A., ... Wahn, B. (2017). Joint action: Mental representations, shared information and general 
mechanisms for coordinating with others. Frontiers in Psychology, 7, 1-7. doi:10.3389/fpsyg.2016.02039

Voltmer, K., \& Von Salisch, M. (2017). Three meta-analyses of children's emotion knowledge and their school success. Learning and Individual Differences, 59, 107118. doi:10.1016/j.lindif.2017.08.006

Warneken, F., Chen, F., \& Tomasello, M. (2006). Cooperative activities in young children and chimpanzees. Child Development, 77(3), 640-663. doi:10.1111/j.14678624.2006.00895.x

Wellman, H. M. (2018). Theory of mind: The state of the art. European Journal of Developmental Psychology, 15(6), 728-755, doi:10.1080/17405629.2018.1435413

Zapiti, A., \& Psaltis, C. (2012). Asymmetries in peer interaction: The effect of social representations of gender and knowledge asymmetry on children's cognitive development. European Journal of Social Psychology, 42, 578-588.

doi:10.1002/ejsp.1885 
EMOTIONS AND PEER ACTION COORDINATION

\section{List of figures}

Figure 1. The labyrinth ball game. 
Table 1. Summary of hierarchical regression analysis for variables predicting peer action coordination

\begin{tabular}{|c|c|c|c|c|c|c|}
\hline \multirow[b]{2}{*}{ Variable } & \multicolumn{3}{|c|}{ Model 1} & \multicolumn{3}{|c|}{ Model 2} \\
\hline & $B$ & $S E B$ & $\beta$ & $B$ & $S E B$ & $\beta$ \\
\hline Age & 4.98 & 1.0 & $0.49 * *$ & 0.10 & 0.02 & $0.38 *$ \\
\hline Gender & -1.5 & 1.0 & -.16 & -1.15 & 0.98 & -.11 \\
\hline Individual SS & 0.18 & 0.06 & $0.27 * *$ & 0.18 & 0.06 & $0.27 * *$ \\
\hline TEC & & & & 0.77 & 0.31 & $0.26^{*}$ \\
\hline$R^{2}$ & & .38 & & & .43 & \\
\hline$F$ for change in $R^{2}$ & & $12.91 *=$ & & & $6.05^{*}$ & \\
\hline
\end{tabular}

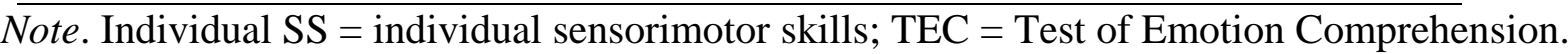
Boys $=0 ;$ Girls $=1$

$* p<.05 . * * p<.001$ 